J. Nonlinear Var. Anal. 4 (2020), No. 3, pp. 415-426

Available online at http://jnva.biemdas.com

https://doi.org/10.23952/jnva.4.2020.3.06

\title{
FIXED POINT RESULTS WITH APPLICATIONS TO INVOLUTION MAPPINGS
}

\author{
LUONG V. NGUYEN*, NGUYEN T.N. TRAM \\ Department of Narural Sciences, Hong Duc University, Thanh Hoa, Vietnam
}

\begin{abstract}
In this paper, we first introduce a class of functions with the property $(L)$, which is weaker than lower semicontinuity property. We then prove a general result on the existence of fixed points of a mapping $T$ in a complete metric $(X, d)$ under the assumption that the function $x \rightarrow d(x, T x)$ has the property. This result generalizes several results in the literature. Finally, we apply our result to the existence of fixed points of involution mappings. Examples are given to illustrate our results.
\end{abstract}

Keywords. Fixed points; Involution mappings; Property (L); Convex metric spaces.

\section{INTRODUCTION AND PRELIMINARIES}

Let $(X, d)$ be a metric space and $K$ be a nonempty subset of $K$. A mapping $T: K \rightarrow K$ is said to be $k$ - Lipschitz if

$$
d(T x, T y) \leq k d(x, y), \quad \forall x, y \in K .
$$

In 1996, Górnicki [3] proved the following result.

Lemma 1.1. [3, Lemma 1] Let $C$ be a nonempty closed convex subset of a Banach space ( $X, \| \cdot$ I) with metric induced by norm $d(x, y)=\|x-y\|$ for all $x, y \in X$ and let $T: C \rightarrow C$ be a $k$ Lipschitz. Assume that there exist constants $a, b \in \mathbb{R}$ with $0 \leq a<1$ and $b>0$ such that for each $x \in C$, there exists $u \in C$ satisfying

(a1) $d(u, T u) \leq \operatorname{a.d}(x, T x)$,

(a2) $d(x, u) \leq b \cdot d(x, T x)$.

Then, $T$ has at least one fixed point in $C$.

This result was restated and proved in the context of convex metric spaces in [1] by Beg (see [1, Theorem 3.1]). We note that the convexity does not play any role in the proofs of the mentioned results. Recently, Górnicki [4] replaced the Lipschitz condition by a condition of Kannan type and proved the following result.

Lemma 1.2. [4, Lemma 1.2] Let $C$ be a nonempty closed subset of a complete metric space $(X, d)$ and let $T: C \rightarrow C$ be a mapping such that there exists $0 \leq K<1$ satisfying

$$
d(T x, T y) \leq K[d(x, T x)+d(y, T y)], \quad \forall x, y \in C .
$$

${ }^{*}$ Corresponding author.

E-mail addresses: luonghdu@gmail.com; nguyenvanluong@hdu.edu.vn(L.V. Nguyen), tramtram2508@gmail. com (D.T.N. Tram).

Received December 10, 2019; Accepted June 2, 2020.

(C)2020 Journal of Nonlinear and Variational Analysis 
Assume that there exist constants $a, b \in \mathbb{R}$ such that $0 \leq a<1$ and $b>0$. If for arbitrary $x \in C$ there exists $u \in C$ such that (a1) and (a2) hold, then $T$ has at least one fixed point.

The following example shows that the Lipschitzianity of $T$ or condition (1.1) is too strong for the existences of a fixed point of $T$.

Example 1.1. Let $X=[-2,10]$ with the usual metric $d(x, y)=|x-y|$ for all $x, y \in X$. Let $T: X \rightarrow X$ be defined as

$$
T x=\left\{\begin{array}{cc}
x / 2, & \text { if }-1<x \leq 10, \\
-8 x-6, & \text { if }-2 \leq x \leq-1 .
\end{array}\right.
$$

We can also check that, for any $x \in X$, there exists $y=0$ such that above conditions (a1) and (a2) hold with $a=1 / 2$ and $b=2$. We also see that $z=0$ is a fixed point of $T$. However, $T$ is not Lipschitz and it does not satify condition (1.1) since $d(T 10, T 0)=5=d(10, T 10)+d(0, T 0)$. Thus, we cannot apply Lemma 1.1, Lemma 1.2 and [1, Theorem 3.1] to this example.

Another example shows that conditions (a1) and (a2) are still strong for the existence of a fixed point of the consideration mapping.

Example 1.2. Let $X=\{0\} \cup\{1 / n: n=1,2, \cdots\}$ with metric $d(x, y)=|x-y|$ for all $x, y \in X$. Then $(X, d)$ is a complete metric space. Consider $T: X \rightarrow X$ defined as

$$
T x=\left\{\begin{array}{cl}
0, & \text { if } x=0, \\
1 /(n+1), & \text { if } x=1 / n, n=1,2, \cdots .
\end{array}\right.
$$

One can easily check that $T$ is $k$-Lipschitz with $k=1$. Moreover, $T$ has a fixed point $z=0$ in $X$. However, conditions (a1) and (a2) are not satisfied. Otherwise, there exist $a \in[0,1)$ and $b>0$ such that for $x=1 / n$ with $n$ sufficiently large, there exists $u \in X$ such that

$$
|u-T u| \leq \frac{a}{n(n+1)},
$$

and

$$
\left|u-\frac{1}{n}\right| \leq \frac{b}{n(n+1)} .
$$

We see that $u$ cannot be 0 when $n$ large enough. Indeed, if $u=0$, then (1.3) becomes $1 \leq$ $b /(n+1)$, which is impossible when $n$ sufficiently large. Hence, $u=1 / m$ with $m \geq 1$ and $m$ depends on $n$. Then (1.2) and (1.3) become

$$
\frac{n(n+1)}{m(m+1)} \leq a
$$

and

$$
\left|1-\frac{n}{m}\right| \leq \frac{b}{n+1}
$$

Since $a<1$, it follows from (1.4 that $n<m$ and $\gamma:=\limsup _{n \rightarrow \infty} \frac{n}{m}<1$. Taking the limit in both sides of (1.5), we obtain $|1-\gamma| \leq 0$, which is a contradiction. Therefore, conditions (a1) and (a2) are not satisfied.

Motivated by above examples, in this paper, we first introduce a class of functions having a property, which will be called Property $(L)$. This class of functions contains the function $x \rightarrow d(x, T x)$ in both examples 1.1 and 1.2 as well as in the case $T$ is Lipschitz or $T$ satisfies (1.1). We then present a fixed point result (Theorem 3.1) for a mapping $T: X \rightarrow X$ under, 
among others, assumption that the function $x \rightarrow d(x, T x)$ having Property $(L)$. Moreover, we replace conditions (a1) and (a2) by more general conditions. So that Theorem 3.1 generalizes both Lemma 1.1 and Lemma 1.2. We note that Lemma 1.1, Lemma 1.2 and [1, Theorem 3.1] were used to study the existence of fixed points of involution mappings in Banach spaces (see, $[3,4]$ ) and in convex metric spaces (see [1]). Following these ideas, in this paper, we also apply Theorem 3.1 to study the existence of fixed points of more general involution mappings in convex metric spaces. For our purpose, the rest of this section will be devoted to recalling some basic definitions about convex metric spaces.

Definition 1.1. [10] Let $(X, d)$ be a metric space. A mapping $W: X \times X \times[0,1] \rightarrow X$ is said to be a convex structure on $X$ if, for each $(x, y, \lambda) \in X \times X \times[0,1]$ and $u \in X$, it holds $d(u, W(x, y, \lambda)) \leq$ $\lambda d(u, x)+(1-\lambda) d(u, y)$. Metric space $(X, d)$ together with the convex structure $W$ is called a convex metric space and is denoted by $(X, d, W)$. A nonempty subset $C$ of a convex metric space $(X, d, W)$ is said to be convex if $W(x, y, \lambda) \in C$ for all $x, y \in C$ and $\lambda \in[0,1]$.

All normed spaces and their convex subsets are convex metric spaces. However, there have many examples of convex metric spaces, which are not embedded in any normed space (see [10]).

Definition 1.2. [2] A convex metric space $(X, d, W)$ is said to have Property $(B)$ if $d(W(x, y, t)$, $W(z, y, t)) \leq t d(x, z)$, for all $x, y, z \in X$ and $t \in(0,1)$.

Remark 1.1. [1, Remark 1.7] Let $(X, d, W)$ be an uniformly convex metric space. If $d(x, z)=a$, $d(y, z)=b$ and $d(x, y)=a+b$, then $z=W(x, y, b /(a+b))$.

Definition 1.3. [9] A convex metric space $(X, d, W)$ is said to be uniformly convex if for any $\varepsilon>0$, there exists $\alpha=\alpha(\varepsilon)$ such that, for all $r>0$ and $x, y, z \in X$ with $d(z, x) \leq r, d(z, y) \leq r$ and $d(x, y) \geq r \varepsilon$, it holds $d(z, W(x, y, 1 / 2)) \leq r(1-\alpha)<r$.

\section{FUnCTIONS HAVING PROPERTY $(L)$}

In this section, we introduce a class of functions having Property $(L)$.

Definition 2.1. Let $(X, d)$ be a metric space and $f: X \rightarrow \mathbb{R}$ be a given function. The function $f$ is said to have Property $(L)$ at a point $x_{0} \in X$ if

$$
\liminf _{x \rightarrow x_{0}} f(x) \leq 0 \Rightarrow f\left(x_{0}\right) \leq 0 .
$$

The function $f$ is said to have property $(L)$ in a subset $K$ of $X$ if it has Property $(L)$ at each $x \in K$.

Remark 2.1. Let $f: X \rightarrow \mathbb{R}$ and $x_{0} \in X$. If $\liminf _{x \rightarrow x_{0}} f(x)>0$, then $f$ has Property $(L)$ at $x_{0}$. If it is lower semicontinuous at $x_{0}$, then it has Property $(L)$ at $x_{0}$.

The following example shows that there are functions having Property $(L)$ but not lower semicontinuous.

Example 2.1. Let $f: \mathbb{R} \rightarrow \mathbb{R}$ be defined by

$$
f(x)=\left\{\begin{array}{cl}
x-1, & \text { if } \quad x<0 \\
x^{2}, & \text { if } \quad x \geq 0
\end{array}\right.
$$


Then, $f$ is not lower semicontinuous at 0 but $f$ has Property $(L)$ at 0 as $\liminf _{x \rightarrow 0} f(x)=-1<$ $0=f(0)$.

Example 2.2. Let $f: \mathbb{R} \rightarrow \mathbb{R}$ be defined by

$$
f(x)= \begin{cases}x-1, & \text { if } \quad x<0 \\ x^{2}+1, & \text { if } \quad x \geq 0\end{cases}
$$

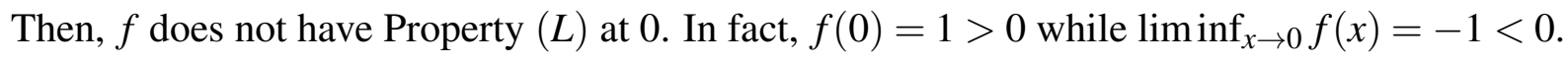

Example 2.3. Let $X, d$ and $T$ be as in Example 1.1. For all $x \in X$, we have

$$
d(x, T x)=\left\{\begin{array}{cc}
|x| / 2, & \text { if }-1<x \leq 10 \\
-9 x-6, & \text { if }-2 \leq x \leq-1
\end{array}\right.
$$

It is obvious that the function $x \rightarrow d(x, T x)$ has Property (L) at any $x \in X$ with $x \neq-1$ since it is continuous at those points. The function $x \rightarrow d(x, T x)$ also has Property $(L)$ at $x=-1$ since $\liminf _{x \rightarrow-1} d(x, T x)=1 / 2>0$. Thus, the function $x \rightarrow d(x, T x)$ has Property $(L)$ in $X$. However, $x \rightarrow d(x, T x)$ is not lower semicontinuous at $x=-1$ since $\liminf _{x \rightarrow-1} d(x, T x)=$ $1 / 2<3=d(-1, T(-1))$.

Example 2.4. Let $(X, d)$ be a metric space and $T: X \rightarrow X$ be a mapping. Let there exist nonnegative numbers $a_{1}, a_{3}, a_{2}, a_{4}, a_{5}$ with $\min \left\{a_{2}+a_{4}, a_{2}+a_{5}, a_{3}+a_{4}, a_{3}+a_{5}\right\}<1$ such that, for all $x, y \in X$,

$$
d(T x, T y) \leq a_{1} d(x, y)+a_{2} d(x, T x)+a_{3} d(y, T y)+a_{4} d(x, T y)+a_{5} d(y, T x) .
$$

Let $f: X \rightarrow \mathbb{R}$ be defined by $f(x)=d(x, T x)$ for all $x \in X$. Then $f$ has Property $(L)$ in $X$. Indeed, let $x \in X$ be such that $\liminf _{y \rightarrow x} f(x) \leq 0$. Then there exists a sequence $\left\{x_{n}\right\}$ in $X$ converging to $x$ such that $\liminf _{n \rightarrow \infty} f\left(x_{n}\right) \leq 0$. Without loss of generality, we assume that $a_{2}+a_{4}<1$. Then

$$
\begin{aligned}
d(x, T x) \leq & d\left(x, x_{n}\right)+d\left(x_{n}, T x_{n}\right)+d\left(T x_{n}, T x\right) \\
\leq & a_{1} d\left(x, x_{n}\right)+a_{2} d(x, T x)+a_{3} d\left(x_{n}, T x_{n}\right)+a_{4} d\left(x_{n}, T x\right) \\
& +a_{5} d\left(x, T x_{n}\right)+d\left(x, x_{n}\right)+d\left(x_{n}, T x_{n}\right) \\
\leq & a_{1} d\left(x, x_{n}\right)+a_{2} d(x, T x)+a_{3} d\left(x_{n}, T x_{n}\right)+a_{4} d\left(x_{n}, x\right)+a_{4} d(x, T x) \\
& +a_{5} d\left(x, x_{n}\right)+a_{5} d\left(x_{n}, T x_{n}\right)+d\left(x, x_{n}\right)+d\left(x_{n}, T x_{n}\right) .
\end{aligned}
$$

It follows that

$$
\frac{1-a_{2}-a_{4}}{1+a_{3}+a_{5}} d(x, T x) \leq \frac{1+a_{1}+a_{4}+a_{5}}{1+a_{3}+a_{5}} d\left(x_{n}, x\right)+d\left(x_{n}, T x_{n}\right),
$$

or, equivalently,

$$
\frac{1-a_{2}-a_{4}}{1+a_{3}+a_{5}} f(x) \leq \frac{1+a_{1}+a_{4}+a_{5}}{1+a_{3}+a_{5}} d\left(x_{n}, x\right)+f\left(x_{n}\right) .
$$

Taking the limit, we obtain

$$
\frac{1-a_{2}-a_{4}}{1+a_{3}+a_{5}} f(x) \leq \liminf _{n \rightarrow \infty} f\left(x_{n}\right) \leq 0 .
$$

Hence, $f(x) \leq 0$ as $0<\left(1-a_{2}-a_{4}\right) /\left(1+a_{3}+a_{5}\right)$. Therefore, $f$ has Property $(L)$ at $x$. 
Example 2.5. Denote by $\Theta$ the set of all functions $\theta:[0, \infty) \rightarrow[0,2)$ satisfying

$$
\limsup _{n \rightarrow \infty} \theta\left(t_{n}\right)<2
$$

for any convergent sequence $\left\{t_{n}\right\} \subset[0, \infty)$.

Let $(X, d)$ be a metric space and $T: X \rightarrow X$. Assume that there exists $\theta \in \Theta$ such that

$$
\begin{aligned}
d(T x, T y) & \leq \theta(\min \{d(x, T x), d(y, T y)\}) \\
& \times \max \left\{d(x, y), \frac{1}{2} d(x, T x), \frac{1}{2} d(y, T y), \frac{1}{2} d(x, T y), \frac{1}{2} d(y, T x)\right\},
\end{aligned}
$$

for all $x, y \in X$. Let $f: X \rightarrow \mathbb{R}$ be defined by $f(x)=d(x, T x)$ for all $x \in X$. Then, $f$ has Property $(L)$ in $X$. Indeed, let $x \in X$ be such that $\liminf _{y \rightarrow x} f(x) \leq 0$. Then, there exists a sequence $\left\{x_{n}\right\}$ in $X$ converging to $x$ and $\lim _{n \rightarrow \infty} f\left(x_{n}\right) \leq 0$. It implies that $\lim _{n \rightarrow \infty} f\left(x_{n}\right)=0$. We show that $f(x)=d(x, T x) \leq 0$. Assume to the contrary that $f(x)>0$. We may assume that $f\left(x_{n}\right)<f(x)$ for all $n$. Since $\left\{f\left(x_{n}\right)\right\}$ converges, by (2.1), there exists $q \in(0,2)$ such that $\limsup _{n \rightarrow \infty} \varphi\left(f\left(x_{n}\right)\right) \leq q$. Then

$$
\begin{aligned}
d(x, T x) \leq & d\left(x, x_{n}\right)+d\left(x_{n}, T x_{n}\right)+d\left(T x_{n}, T x\right) \\
\leq & d\left(x, x_{n}\right)+d\left(x_{n}, T x_{n}\right)+\theta\left(\min \left\{d(x, T x), d\left(x_{n}, T x_{n}\right)\right\}\right) \\
& \quad \times \max \left\{d\left(x, x_{n}\right), \frac{1}{2} d(x, T x), \frac{1}{2} d\left(x_{n}, T x_{n}\right), \frac{1}{2} d\left(x, T x_{n}\right), \frac{1}{2} d\left(x_{n}, T x\right)\right\} \\
\leq & q \max \left\{d\left(x, x_{n}\right), \frac{1}{2} d(x, T x), \frac{1}{2} d\left(x_{n}, T x_{n}\right),\right. \\
& \left.\quad \frac{1}{2} d\left(x, x_{n}\right)+\frac{1}{2} d\left(x_{n}, T x_{n}\right), \frac{1}{2} d\left(x_{n}, x\right)+\frac{1}{2} d(x, T x)\right\} \\
& +d\left(x, x_{n}\right)+d\left(x_{n}, T x_{n}\right) \\
\leq & \frac{q}{2}\left[2 d\left(x, x_{n}\right)+d(x, T x)+d\left(x_{n}, T x_{n}\right)\right]+d\left(x, x_{n}\right)+d\left(x_{n}, T x_{n}\right) .
\end{aligned}
$$

Hence,

$$
\frac{2-q}{2+q} d(x, T x) \leq d\left(x_{n}, T x_{n}\right)+\frac{2 q+2}{2+q} d\left(x_{n}, x\right) .
$$

Taking the limit, we get

$$
\frac{2-q}{2+q} d(x, T x) \leq 0
$$

which contradicts the assumption that $d(x, T x)>0$ as $0<(2-q) /(2+q)$. Therefore, $f$ has Property $(L)$ at $x$.

\section{FIXED POINT RESULTS}

This section is devoted to fixed point results. Before stating our first fixed point result, we introduce some classes of functions. Let $f: X \rightarrow[0, \infty)$ be a function. We denote by $\mathscr{A}$ the set of all functions $\alpha: X \times X \rightarrow[0,1]$ satisfying: for any sequence $\left\{x_{n}\right\} \subset X$, if $\left\{f\left(x_{n}\right)\right\}$ converges, then

$$
\limsup _{n \rightarrow \infty} \alpha\left(x_{n}, x_{n+1}\right)<1 \text {. }
$$


We denote by $\mathscr{K}(f)$ the set of functions $\kappa: X \times X \rightarrow[0, \infty)$ satisfying: for any sequence $\left\{x_{n}\right\} \subset$ $X$, if $\left\{f\left(x_{n}\right)\right\}$ is a nonincreasing sequence converging to 0 , then the sequence $\left\{\kappa\left(x_{n}, x_{n+1}\right)\right\}$ is bounded. We also denote by $\mathscr{L}(f)$ the set of all functions $\ell: X \times X \rightarrow[0, \infty)$ satisfying: for any sequence $\left\{x_{n}\right\} \subset X$, if $\left\{f\left(x_{n}\right)\right\}$ is a nonincreasing sequence converging to 0 , then the sequence $\left\{\ell\left(x_{n}, x_{n+1}\right)\right\}$ is bounded below away from zero, i.e., there exists $M>0$ such that $\ell\left(x_{n}, x_{n+1}\right) \geq M$ for all $n$.

Example 3.1. Let $(X, d)$ be a metric space and $f: X \rightarrow[0, \infty)$ be given function. Assume that $a, b, c$ are positive numbers satisfying $a \in(0,1), b>0$ and $c>0$. Let $\alpha: X \times X \rightarrow[0,1]$, $\kappa: X \times X \rightarrow[0, \infty)$ and $\ell: X \times X \rightarrow[0, \infty)$ be defined by: $\alpha(x, y)=a, \kappa(x, y)=b$ and $\ell(x, y)=c$ for all $x, y \in X$. Then $\alpha \in \mathscr{A}(f), \kappa \in \mathscr{K}(f)$ and $\ell \in \mathscr{L}(f)$.

Example 3.2. Let $(X, d)$ be a metric space and $f: X \rightarrow[0, \infty)$ be given function. Let $\alpha$ : $X \times X \rightarrow[0,1] \kappa: X \times X \rightarrow[0, \infty)$ and $\ell: X \times X \rightarrow \mathbb{R}$ be defined by: for all $x, y \in X$

$$
\alpha(x, y)=\frac{f(x)}{f(x)+f(y)+1}, \quad \kappa(x, y)=f(x) \cdot f(y)+5
$$

and

$$
\ell(x, y)=\sqrt{f(x)+f(y)+2} .
$$

Then $\alpha \in \mathscr{A}(f), \kappa \in \mathscr{K}(f)$ and $\ell \in \mathscr{L}(f)$.

Our first fixed point theorem is stated as follows.

Theorem 3.1. Let $(X, d)$ be a complete metric space and $T: X \rightarrow X$. Define $f: X \rightarrow[0, \infty)$ by $f(x)=d(x, T x)$ for all $x \in X$. Assume that there exist $\alpha \in \mathscr{A}(f), \kappa \in \mathscr{K}(f)$ and $\ell \in \mathscr{L}(f)$ such that for each $x \in X$, there exists $y \in X$ satisfying

$$
d(y, T y) \leq \alpha(x, y) d(x, T x),
$$

and

$$
d(x, y) \leq \kappa(x, y)[d(x, T x)]^{\ell(x, y)} .
$$

Then, $T$ has at least one fixed point in $X$ provided that the function $f$ has Property $(L)$ in $X$.

Proof. Let $x_{0} \in X$. Under the assumptions, we can construct a sequence $\left\{x_{n}\right\}$ in $X$ satisfying the following conditions: for all $n$,

$$
d\left(x_{n+1}, T x_{n+1}\right) \leq \alpha\left(x_{n}, x_{n+1}\right) d\left(x_{n}, T x_{n}\right),
$$

and

$$
d\left(x_{n}, x_{n+1}\right) \leq \kappa\left(x_{n}, x_{n+1}\right)\left[d\left(x_{n}, T x_{n}\right)\right]^{\ell\left(x_{n}, x_{n+1}\right)} .
$$

Since $\alpha(x, y) \in[0,1]$ for all $x, y \in X$, from (3.3), one has

$$
d\left(x_{n+1}, T x_{n+1}\right) \leq d\left(x_{n}, T x_{n}\right) \text { for all } n .
$$

Hence $\left\{d\left(x_{n}, T x_{n}\right)\right\}$ is a nonincreasing sequence of nonnegative real numbers. Thus, there exists $\gamma \geq 0$ such that

$$
\lim _{n \rightarrow \infty} f\left(x_{n}\right)=\lim _{n \rightarrow \infty} d\left(x_{n}, T x_{n}\right)=\gamma .
$$

Since $\alpha \in \mathscr{A}(f)$, there exists $\beta \in(0,1)$ such that

$$
\beta=\limsup _{n \rightarrow \infty} \alpha\left(x_{n}, x_{n+1}\right) \text {. }
$$


Assume that $\gamma>0$. Taking the limit on both sides of (3.3), we obtain that

$$
\gamma=\underset{n \rightarrow \infty}{\limsup } d\left(x_{n+1}, T x_{n+1}\right) \leq \limsup _{n \rightarrow \infty} \alpha\left(x_{n}, x_{n+1}\right) d\left(x_{n}, T x_{n}\right) \leq \beta \gamma<\gamma,
$$

which is a contradiction. Hence, $\gamma=0$, i.e.,

$$
\lim _{n \rightarrow \infty} f\left(x_{n}\right)=\lim _{n \rightarrow \infty} d\left(x_{n}, T x_{n}\right)=0 .
$$

Since $\kappa \in \mathscr{K}(f)$ and $\ell \in \mathscr{L}(f)$, there exist $K>0$ and $L>0$ such that $\kappa\left(x_{n}, x_{n+1}\right) \leq K$ and $\ell\left(x_{n}, x_{n+1}\right) \geq L$ for all $n$. Now, let $\delta \in(\beta, 1)$. By (3.5) and (3.6), there exists $n_{0} \in \mathbb{N}$ such that

$$
\alpha\left(x_{n}, x_{n+1}\right)<\delta \text { and } f\left(x_{n}\right)<1,
$$

for all $n \geq n_{0}$. From (3.3), we have

$$
d\left(x_{n+1}, T x_{n+1}\right)<\delta d\left(x_{n}, T x_{n}\right), \quad \text { for all } n \geq n_{0} .
$$

Then, by induction, we get

$$
d\left(x_{n}, T x_{n}\right)<\delta^{n-n_{0}} d\left(x_{n_{0}}, T x_{n_{0}}\right), \text { for all } n>n_{0} .
$$

Using the latter inequality, (3.4) and the fact that $d\left(x_{n}, T x_{n}\right)<1$ whenever $n>n_{0}$, we have

$$
\begin{aligned}
d\left(x_{n}, x_{n+1}\right) & \leq \kappa\left(x_{n}, x_{n+1}\right)\left[d\left(x_{n}, T x_{n}\right)\right]^{\ell\left(x_{n}, x_{n+1}\right)} \leq K\left[d\left(x_{n}, T x_{n}\right)\right]^{\ell\left(x_{n}, x_{n+1}\right)} \\
& \leq K\left[d\left(x_{n}, T x_{n}\right)\right]^{L}<K\left[\delta^{n-n_{0}} d\left(x_{n_{0}}, T x_{n_{0}}\right)\right]^{L} \\
& =K\left[d\left(x_{n_{0}}, T x_{n_{0}}\right)\right]^{L} \delta^{L\left(n-n_{0}\right)}, \quad \text { for all } n>n_{0} .
\end{aligned}
$$

Since $\delta \in(0,1)$, we have, for $m>n>n_{0}$, that

$$
\begin{aligned}
d\left(x_{n}, x_{m}\right) & \leq d\left(x_{n}, x_{n+1}\right)+\cdots+d\left(x_{m-1}, x_{m}\right) \\
& \leq K\left[d\left(x_{n_{0}}, T x_{n_{0}}\right)\right]^{L} \delta^{L\left(n-n_{0}\right)}+\cdots+K\left[d\left(x_{n_{0}}, T x_{n_{0}}\right)\right]^{L} \delta^{L\left(m-1-n_{0}\right)} \\
& \leq K\left[d\left(x_{n_{0}}, T x_{n_{0}}\right)\right]^{L} \delta^{L\left(n-n_{0}\right)}\left(1+\delta^{L}+\delta^{2 L}+\cdots\right) \\
& =\frac{K\left[d\left(x_{n_{0}}, T x_{n_{0}}\right)\right]^{L}}{1-\delta^{L}} \delta^{L\left(n-n_{0}\right)}
\end{aligned}
$$

This implies that $\left\{x_{n}\right\}$ is a Cauchy sequence. Since $X$ is complete, there exists $z \in X$ such that $\left\{x_{n}\right\}$ converges to $z$. Since $f$ has property $(L)$, one has $d(z, T z)=f(z) \leq 0$. This implies that $d(z, T z)=0$, i.e., $T z=z$. This ends the proof.

Remark 3.1. Theorem 3.1 includes [3, Lemma 1], [1, Theorem 3.1] and [4, Lemma 1.2] as special cases.

If we replace $\alpha(x, y), \kappa(x, y)$ and $\ell(x, y)$ in Theorem 3.1 by suitable functions, we can get some corollaries which have simpler forms and are easier to apply. For instance,

Corollary 3.1. Let $(X, d)$ be a complete metric space and $T: X \rightarrow X$. Assume that there exist $a \in(0,1), k>0$ and $\ell>0$ such that, for each $x \in X$, there exists $y \in X$ satisfying

$$
d(y, T y) \leq a d(x, T x),
$$

and

$$
d(x, y) \leq k[d(x, T x)]^{\ell} .
$$


Then, $T$ has at least one fixed point in $X$ provided that the function $d(x, T x)$ has property $(L)$ in $X$.

Example 3.3. Let $X, d$ and $T$ be as in Example 1.1. Then the function $x \rightarrow d(x, T x)$ has property $(L)$ in $X$. We can also check that, for any $x \in X$, there exists $y=0$ such that (3.7) and (3.8) hold with $\alpha=1 / 2, \ell=1$ and $k=2$. Thus, all assumptions of Corollary 3.1 are satisfied and $z=0$ is a fixed point of $T$. However, $T$ is not Lipschitz and it does not satisfy condition (1.1). Thus, we cannot apply [3, Lemma 1], [1, Theorem 3.1] and [4, Lemma 1.2] to this example.

The following example shows the significance of the power $\ell(x, y)$ when it is not equal to 1 .

Example 3.4. Let $X, d$ and $T$ be as in Example 1.2. As in Example 1.2, we cannot apply [3, Lemma 1], [1, Theorem 3.1] and [4, Lemma 1.2] to this example since conditions (a1) and (a2) are not satisfied. However, we can apply Corollary 3.1 to this example. Indeed, we have

$$
d(x, T x)=\left\{\begin{array}{cl}
0, & \text { if } x=0, \\
\frac{1}{n(n+1)}, & \text { if } x=\frac{1}{n}, n=1,2, \cdots .
\end{array}\right.
$$

It is evident that the function $x \rightarrow d(x, T x)$ is lower semicontinuous and hence it has Property $(L)$ in $X$. Moreover, there exist $a=1 / 2, k=2$ and $\ell=1 / 2$ such that, for each $x \in X$, there exists $y=0$ satisfying (3.7) and (3.8). It is obvious when $x=0$. If $x=1 / n, n=1,2, \cdots$, then we can easily see that (3.7) holds and (3.8) is equivalent to

$$
\frac{1}{n} \leq 2\left(\frac{1}{n(n+1)}\right)^{1 / 2}
$$

or, equivalently,

$$
1 \leq \frac{4 n}{n+1}
$$

which obviously holds for $n \geq 1$. Thus, all assumptions of Corollary 3.1 are satisfied.

We next present some applications of Theorem 3.1. We first apply Theorem 3.1 to give another shorter proof for the main result in [8].

Theorem 3.2. [8, Theorem 2.4] Let $(X, d)$ be a complete metric space and $T: X \rightarrow X$ be a mapping such that, for all $x, y \in X$,

$$
d(T x, T y) \leq a d(x, y)+b d(x, T x)+c d(y, T y)+e d(y, T x)+f d(x, T y),
$$

where $0<a<1, b, c, e, f \geq 0, b+c>0$ and $a+b+c+e+f=1$. If $e+f>0$, then $T$ has $a$ unique fixed point.

Proof. Let $x \in X$ and set $z=T^{2} x$. As in Step 1 of the proof of Theorem 2.4 in [8], there exists $\lambda \in(0,1)$ such that

$$
d(z, T z) \leq \lambda d(x, T x) .
$$

Moreover,

$$
\begin{aligned}
d(x, z) & =d\left(x, T^{2} x\right) \leq d(x, T x)+d\left(T x, T^{2} x\right) \\
& \leq d(x, T x)+a d(x, T x)+b d(x, T x)+c d\left(T x, T^{2} x\right)+e d(T x, T x)+f d\left(x, T^{2} x\right) \\
& =(1+a+b) d(x, T x)+c d(T x, z)+f d(x, z) \\
& \leq(1+a+b+c) d(x, T x)+(c+f) d(x, z) .
\end{aligned}
$$


This implies that

$$
d(x, z) \leq \frac{1+a+b+c}{1-c-f} d(x, T x) .
$$

Since the function $x \rightarrow d(x, T x)$ has Property $(L)$, the existence of a fixed point of $T$ follows Corollary 3.1. The uniqueness of fixed point of $T$ follows from inequality (3.9).

Corollary 3.2. Let $(X, d)$ be a complete metric space and $T: X \rightarrow X$. Assume that, for all $x, y \in X$,

$$
d\left(T^{2} x, T^{2} y\right) \leq a d(x, y)+b d\left(x, T^{2} x\right)+c d\left(y, T^{2} y\right)+e d\left(y, T^{2} x\right)+f d\left(x, T^{2} y\right)
$$

with $a, b, c, e$ and $f$ as in Theorem 3.2. Then, $T$ has a unique fixed point in $X$.

Proof. By Theorem 3.2, $T^{2}$ has a unique fixed point, say $x$, in $X$. Then

$$
\begin{aligned}
d(x, T x) & =d\left(T^{2} x, T^{2}(T x)\right) \\
& \leq a d(x, T x)+b d\left(x, T^{2} x\right)+c d\left(T x, T^{3} x\right)+e d\left(T x, T^{2} x\right)+f d\left(x, T^{3} x\right) \\
& =(a+e+f) d(x, T x),
\end{aligned}
$$

which implies that $(1-a-e-f) d(x, T x) \leq 0$. Thus, $d(x, T x)=0$, i.e., $x$ is a fixed point of $T$. Assume that $z$ is another fixed point of $T$. Then, $T^{2} z=T z=z$, which implies that $z=x$. This ends the proof.

We next give an example for which Theorem 3.2 cannot be applied but Corollary 3.2 can.

Example 3.5. Let $X=\mathbb{R}$ with the usual metric $d(x, y)=|x-y|$ for all $x, y \in X$. Consider the mapping $T: \mathbb{R} \rightarrow \mathbb{R}$ defined as

$$
T x= \begin{cases}-5 x & \text { if } x \geq 0 \\ -\frac{1}{10} x & \text { if } x<0\end{cases}
$$

Then, for $x \geq 0$, we have $T^{2} x=T(-5 x)=-(-5 x) / 10=x / 2$, and for $x<0$, we get $T^{2} x=$ $T(-x / 10)=-5(-x / 10)=x / 2$. Thus, $T^{2} x=x / 2$ for all $x \in X$. It is easy to check that $T$ satisfies (3.10) with $a=1 / 2$ and $b=c=e=f=1 / 8$. Applying Corollary 3.2, we conclude that $T$ has a unique fixed point. In fact, 0 is the unique fixed point of $T$. However, we cannot apply Theorem 3.2 to this example since condition (3.9) in Theorem 3.2 is not satisfied. Otherwise, for $(x, y)=(1,0)$ and $(x, y)=(0,1)$, we should obtain $5 \leq a+6 b+5 e+f$ and $5 \leq a+6 c+e+5 f$. From the last two inequalities, we have $5 \leq a+3(b+c+e+f)$. Since $a+b+c+e+f=1$, the latter inequality yields $2 \leq b+c+e+f$ which is a contradiction.

We next apply Theorem 3.1 to prove the existence of fixed points for a class of involution mappings. Recall that a mapping $T: X \rightarrow X$ is called an involution if $T^{2}=I$ on $X$, where $I$ is the identity mapping on $X$.

Theorem 3.3. Let $(X, d, W)$ be a convex complete metric space, $K$ be a nonempty closed convex subset of $X$ and $T: K \rightarrow K$ be an involution. Assume that there exists $\theta \in \Theta$ such that

$$
\begin{aligned}
d(T x, T y) & \leq \theta(\min \{d(x, T x), d(y, T y)\}) \\
& \times \max \left\{d(x, y), \frac{1}{2} d(x, T x), \frac{1}{2} d(y, T y), \frac{1}{2} d(x, T y), \frac{1}{2} d(y, T x)\right\}
\end{aligned}
$$

for all $x, y \in K$. Then, $T$ has at least one fixed point in $K$. 
Proof. By Example 2.5, we see that $x \rightarrow d(x, T x)$ has property $(L)$. Suppose that $T$ has no fixed point in $K$, i.e., $d(x, T x)>0$ for all $x \in K$. For each $x \in K$, let $y=W(x, T x, 1 / 2)$. Then

$$
d(x, y)=d(x, W(x, T x, 1 / 2)) \leq \frac{1}{2} d(x, x)+\frac{1}{2} d(x, T x)=\frac{1}{2} d(x, T x),
$$

and

$$
d(y, T x)=d(T x, W(x, T x, 1 / 2)) \leq \frac{1}{2} d(T x, x)+\frac{1}{2} d(T x, T x)=\frac{1}{2} d(x, T x) .
$$

Using above inequalities, we have

$$
\begin{aligned}
d(x, T y) & =d\left(T^{2} x, T y\right) \\
\leq & \theta\left(\min \left\{d\left(T x, T^{2} x\right), d(y, T y)\right\}\right) \\
& \times \max \left\{d(T x, y), \frac{1}{2} d\left(T x, T^{2} x\right), \frac{1}{2} d(y, T y), \frac{1}{2} d(T x, T y), \frac{1}{2} d\left(y, T^{2} x\right)\right\} \\
\leq & \theta(\min \{d(x, T x), d(y, T y)\}) \\
& \times \max \left\{d(y, T x), \frac{1}{2} d(x, T x), \frac{1}{2} d(y, T y), \frac{1}{2} d(T x, T y), \frac{1}{2} d(x, y)\right\} \\
& \leq \frac{1}{2} \theta(\min \{d(x, T x), d(y, T y)\}) \cdot \max \{d(x, T x), d(y, T y), d(T x, T y)\},
\end{aligned}
$$

and

$$
\begin{aligned}
d(T x, T y) & \leq \theta(\min \{d(x, T x), d(y, T y)\}) \\
& \times \max \left\{d(x, y), \frac{1}{2} d(x, T x), \frac{1}{2} d(y, T y), \frac{1}{2} d(x, T y), \frac{1}{2} d(y, T x)\right\} \\
& \leq \frac{1}{2} \theta(\min \{d(x, T x), d(y, T y)\}) \cdot \max \{d(x, T x), d(y, T y), d(x, T y)\} .
\end{aligned}
$$

Assume that $d(T x, T y) \geq \max \{d(x, T x), d(y, T y)\}$. It follows from (3.12) that

$$
d(x, T y) \leq \frac{1}{2} \theta(\min \{d(x, T x), d(y, T y)\}) . d(T x, T y)<d(T x, T y) .
$$

This, together with (3.13), implies that

$$
\begin{aligned}
d(T x, T y) & \leq \frac{1}{2} \theta(\min \{d(x, T x), d(y, T y)\}) \cdot \max \{d(x, T x), d(y, T y)\} \\
& <\max \{d(x, T x), d(y, T y)\},
\end{aligned}
$$

which is a contradiction. Thus, $d(T x, T y)<\max \{d(x, T x), d(y, T y)\}$ and then

$$
d(x, T y) \leq \frac{1}{2} \theta(\min \{d(x, T x), d(y, T y)\}) \cdot \max \{d(x, T x), d(y, T y)\},
$$

and

$$
d(T x, T y) \leq \frac{1}{2} \theta(\min \{d(x, T x), d(y, T y)\}) \cdot \max \{d(x, T x), d(y, T y)\} .
$$

Using (3.14) and (3.15), we have

$$
\begin{aligned}
d(y, T y) & =d(T y, W(x, T x, 1 / 2)) \leq \frac{1}{2} d(T y, x)+\frac{1}{2} d(T y, T x) \\
& \leq \frac{1}{2} \theta(\min \{d(x, T x), d(y, T y)\}) \cdot \max \{d(x, T x), d(y, T y)\},
\end{aligned}
$$


which implies that

$$
d(y, T y) \leq \frac{1}{2} \theta(\min \{d(x, T x), d(y, T y)\}) \cdot d(x, T x) .
$$

Applying Theorem 3.1 with $\alpha(x, y)=\theta(\min \{d(x, T x), d(y, T y)\}) / 2, \kappa(x, y)=1 / 2$ and $\ell(x, y)=$ 1 for all $x, y \in X$, we conclude that $T$ has a fixed point in $K$.

We can omit the assumption that $T^{2}=I$ in the previous result by assuming that $T^{2}$ satisfies a certain contractive condition and that $X$ is uniformly convex, $K$ is bounded. Our next result is based on the following result by Fukhar-ud-din, Khana and Akhtar [6].

Theorem 3.4. [6] Let $K$ be a nonempty, closed, convex and bounded subset of a complete and uniformly convex metric space $(X, d, W)$ having Property $(B)$. If $T: K \rightarrow K$ is a continuous map satisfying (3.9) with $a, b, c, e, f \geq 0$ and $a+b+c+e+f \leq 1$, then $T$ has a fixed point in $K$.

Remark 3.2. Exploiting the proof of Theorem 3.4 in [6], we see that the continuity of $T$ is only used to obtain that the sets $C_{\beta}=\{x: d(x, T x) \leq \beta\}$, where $\beta \in(0,1)$, are closed (see the proof in [6, page 4753]). It is easy to see that the sets $C_{\beta}$ are closed if the function $x \rightarrow f(x):=d(x, T x)$ is lower semicontinuous. Thus, the conclusion of Theorem 3.4 still holds true if we replace the continuity of $T$ by the lower semicontinuity of $f$.

Theorem 3.5. Let $K$ be a nonempty, closed, convex and bounded subset of a complete and uniformly convex metric space $(X, d, W)$ having Property $(B)$ and $T: K \rightarrow K$ be such that $x \rightarrow$ $d\left(x, T^{2} x\right)$ is lower continuous. Assume that there exist $a, b, c, d, e, f \geq 0$ with $a+b+c+d+e+$ $f \leq 1$ such that

$$
d\left(T^{2} x, T^{2} y\right) \leq a d(x, y)+b d\left(x, T^{2} x\right)+c d\left(y, T^{2} y\right)+e d\left(y, T^{2} x\right)+f d\left(x, T^{2} y\right),
$$

for all $x, y \in K$. If, in addition, there exists $\theta \in \Theta$ such that (3.11) holds, then $T$ has a fixed point in $K$.

Proof. We may assume that $b=c, e=f$ and $a+2 b+2 e \leq 1$. Set $C=\left\{x \in K: T^{2} x=x\right\}$. By Theorem 3.4 and Remark 3.2, $C$ is nonempty. We next show that $C$ is closed. Let $\left\{x_{n}\right\}$ be a sequence in $C$ converging to some $x \in X$. By the lower semicontinuity of $x \rightarrow d\left(T^{2} x, x\right)$, we have

$$
0 \leq d\left(T^{2} x, x\right) \leq \liminf _{n \rightarrow \infty} d\left(T^{2} x_{n}, x_{n}\right)=0 .
$$

This implies that $d\left(T^{2} x, x\right)=0$, i.e., $T^{2} x=x$. So, $x \in C$ and thus $C$ is closed. We now prove that $C$ is convex. To this end let $x, y \in C$ and $t \in(0,1)$, we will show that $W(x, y, t) \in C$. Observe that

$$
\begin{aligned}
d\left(x, T^{2}(W(x, y, t))\right)= & d\left(T^{2} x, T^{2}(W(x, y, t))\right) \\
\leq & a d(x, W(x, y, t))+b\left[d\left(x, T^{2} x\right)+d\left(W(x, y, t), T^{2}(W(x, y, t))\right)\right] \\
& +e\left[d\left(x, T^{2}(W(x, y, t))\right)+d\left(W(x, y, t), T^{2} x\right)\right] \\
\leq & (a+b+e) d(x, W(x, y, t))+(b+e) d\left(x, T^{2}(W(x, y, t))\right) .
\end{aligned}
$$

This yields

$$
d\left(x, T^{2}(W(x, y, t))\right) \leq \frac{a+b+c}{1-b-e} d(x, W(x, y, t)) .
$$


Similarly, one has

$$
d\left(y, T^{2}(W(x, y, t))\right) \leq \frac{a+b+e}{1-b-e} d(y, W(x, y, t)) .
$$

Using (3.17) and (3.18), one has

$$
\begin{aligned}
d(x, y) & \leq d\left(x, T^{2}(W(x, y, t))\right)+d\left(y, T^{2}(W(x, y, t))\right) \\
& \leq \frac{a+b+e}{1-b-e}[d(x, W(x, y, t))+d(y, W(x, y, t))] \\
& \leq \frac{a+b+c}{1-b-e}[(1-t) d(x, y)+t d(y, x)]=\frac{a+b+e}{1-b-e} d(x, y) .
\end{aligned}
$$

If $a+2 b+2 e<1$, then it follows from (3.19) that $W(x, y, t)=x=y$. Thus, $W(x, y, t) \in C$. Assume that $a+2 b+2 e=1$. From (3.17) - (3.19). Then

$$
d\left(x, T^{2}(W(x, y, t))\right)=d(x, W(x, y, t))=(1-t) d(x, y),
$$

and

$$
d\left(y, T^{2}(W(x, y, t))\right)=d(y, W(x, y, t))=t d(x, y) .
$$

From (3.20) and (3.21), we have

$$
\frac{d(y, W(x, y, t))}{d(x, W(x, y, t))+d(y, W(x, y, t))}=\frac{t d(x, y)}{d(x, y)}=t .
$$

Then, by Remark $1.1, T^{2}(W(x, y, t))=W(x, y, t)$, that is, $W(x, y, t) \in C$. Thus, $C$ is convex. Moreover, $T(C)=C$ and $T^{2}=I$ on $C$. Therefore, by Theorem 3.3, $T$ has a fixed point in $K$.

Remark 3.3. In the setting of uniformly convex metric spaces, one can improve the assumption on functions $\theta$ based on the modulus of convexity as, e.g., in [1, Theorem 3.4], [5, Theorem 3] and [7, Theorem 2.4] to get a more general result than mentioned results.

\section{REFERENCES}

[1] I. Beg, Inequalities in metric spaces with applications, Topol. Methods Nonlinear Anal. 17 (2001), 183-190.

[2] I. Beg, M. Abbas, Fixed point and best approximation in Menger convex metric spaces, Arch. Math. 41 (2005), 389-397.

[3] J. Górnicki, Fixed points of involutions. Math. Japonica 43 (1996), 151-155.

[4] J. Górnicki, Fixed point theorems for Kannan type mappings, J. Fixed Point Theory Appl. 19 (2017), 21452152.

[5] J. Górnicki, B. E. Rhoades, A general fixed point theorem for involutions, Indian J. Pure Appl. Math. 27 (1996), 13-23.

[6] H. Fukhar-ud-din, A.R. Khana, Z. Akhtar, Fixed point results for a generalized nonexpansive map in uniformly convex metric spaces, Nonlinear Anal. 75 (2012), 4747-4760.

[7] M.S. Khan, M. Imdad, Fixed points of certain involutions in Banach spaces, J. Austral. Math. Soc. (Series A) 37 (1984), $169-177$.

[8] S. Moradi, A. Farajzadeh, On Olaleru's open problem on Gregus fixed point theorem, J. Glob. Optim. 56 (2013), 1689-1697.

[9] T. Shimizu, W. Takahashi, Fixed points of multivalued mappings in certain convex metric spaces, Topol. Methods Nonlinear Anal. 8 (1996), 197-203.

[10] W. Takahashi, A convexity in metric spaces and nonexpansive mapping I, Kodai Math. Sem. Rep. 22 (1970), 142-149. 